\title{
Dynamical analysis of a delayed predator-prey system with modified Leslie-Gower and Beddington-DeAngelis functional response
}

\section{Juan Liu*}

"Correspondence:

liujuan7216@163.com Department of Mathematics and Physics, Bengbu College, Bengbu, 233030, P.R. China

\section{Springer}

\begin{abstract}
The dynamics of a delayed predator-prey system with modified Leslie-Gower and Beddington-DeAngelis functional response is investigated. The main results are given in terms of local stability and local Hopf bifurcation. By regarding the possible combination of the feedback delays of the prey and the predator as a bifurcation parameter, sufficient conditions for the local stability and existence of the local Hopf bifurcation of the system are obtained. In particular, the properties of the local Hopf bifurcation such as direction and stability are determined by using the normal form method and center manifold theorem. Finally, numerical simulations are carried out to illustrate the main theoretical results.
\end{abstract}

Keywords: delays; Hopf bifurcation; predator-prey system; stability; periodic solution

\section{Introduction}

As we all know, one of the dominant themes in mathematical ecology is the dynamic relationship between predators and their prey. One of the important factors which affect the dynamics of biological and mathematical models is the functional response. Especially the Beddington-DeAngelis functional response which is first proposed by Beddington and DeAngelis et al. [1, 2]. Beddington-DeAngelis functional response has desirable qualitative features of ratio dependent form but takes care of their controversial behaviors at low densities and it has an extra term in the denominator modeling mutual interference among predators. Predator-prey systems with a Beddington-DeAngelis functional response have been studied by many authors [3-12]. Ko and Ryu studied a diffusive two-competing prey and one-predator system with Beddington-DeAngelis functional response and discussed the stability and uniqueness of coexistence states [3]. Liu and Wang studied the global asymptotic stability of two stage-structured predator-prey systems with BeddingtonDeAngelis functional response [6]. Li and Takeuchi studied the permanence, local stability, and global stability of two models of a density dependent predator-prey system with Beddington-DeAngelis functional response by using stability theory and Lyapunov functions [10]. Yu investigated the permanence, local stability, and global stability of the following modified Leslie-Gower predator-prey system with Beddington-DeAngelis functional

(c)2014 Liu; licensee Springer. This is an Open Access article distributed under the terms of the Creative Commons Attribution License (http://creativecommons.org/licenses/by/2.0), which permits unrestricted use, distribution, and reproduction in any medium, provided the original work is properly cited. 
response [12]:

$$
\left\{\begin{array}{l}
\frac{d x(t)}{d t}=\left(r_{1}-p x(t)-\frac{\alpha y(t)}{1+b x(t)+c y(t)}\right) x(t), \\
\frac{d y(t)}{d t}=\left(r_{2}-\frac{\beta y(t)}{x(t)+k}\right) y(t),
\end{array}\right.
$$

where $x(t)$ and $y(t)$ represent the densities of the prey and the predator at time $t$, respectively. $r_{1}$ and $r_{2}$ are the intrinsic growth rates of the prey and the predator, respectively. $p$ is the intra-specific competition coefficient of the prey. $\alpha$ is the maximum value of the per capita reduction rate of the prey due to the predator. $\beta$ is a measure of the food quantity that the prey provides converted to the predator birth.

It is well known that dynamical systems with one delay or multiple delays have been studied by many authors $[7,10,11,13-20]$. Li and Wang studied the stability and Hopf bifurcation of a delayed three-level food chain model with Beddington-DeAngelis functional response [7]. Bianca et al. studied the stability and Hopf bifurcation of a mathematical framework that consists of a system of a logistic equation with a delay and an equation for the carrying capacity by regarding the delay as a bifurcation parameter [13]. Cui and Yan studied a three-species food chain system with two delays and they analyzed the Hopf bifurcation of the system by taking the sum of the two delays as the bifurcation parameter [17]. In [20], Bianca et al. further studied an economic growth model with two delays and they investigated the existence and properties of Hopf bifurcation of the model by regarding the possible combination of the two delays as the bifurcation parameter. To the best of our knowledge, seldom did authors consider the Hopf bifurcation of the delayed predatorprey system with modified Leslie-Gower and Beddington-DeAngelis functional response. Stimulated by this, in this paper we investigate the Hopf bifurcation of the following delayed predator-prey system with modified Leslie-Gower and Beddington-DeAngelis functional response:

$$
\left\{\begin{array}{l}
\frac{d x(t)}{d t}=\left(r_{1}-p x\left(t-\tau_{1}\right)-\frac{\alpha y(t)}{1+b x(t)+c y(t)}\right) x(t), \\
\frac{d y(t)}{d t}=\left(r_{2}-\frac{\beta y\left(t-\tau_{2}\right)}{x\left(t-\tau_{2}\right)+k}\right) y(t),
\end{array}\right.
$$

where $\tau_{1} \geq 0$ is the feedback delay of the prey and $\tau_{2} \geq 0$ is the feedback delay of the predator. The main purpose of this paper is to consider the effect of the two delays on the dynamics of system (2).

This paper is organized as follows. In Section 2, we discuss local stability of the positive equilibrium and existence of the local Hopf bifurcation of system (2). In Section 3, direction of the Hopf bifurcation and stability of the bifurcated periodic solutions are determined by using the normal form method and center manifold theorem. In Section 4, some numerical simulations are performed to illustrate our theoretical analysis and a final conclusion is given in Section 5 .

\section{Local stability and the existence of Hopf bifurcation}

It is easy to verify that if the condition $\left(\mathrm{H}_{1}\right): \alpha r_{2} k<r_{1} \beta+r_{1} r_{2} c k$ holds, then system (2) has a unique positive equilibrium $E_{*}\left(x_{*}, y_{*}\right)$, where $y_{*}=\frac{r_{2}\left(x_{*}+k\right)}{\beta}$, and $x_{*}=\frac{-B+\sqrt{B^{2}-4 A C}}{2 A}$, where $A=b p \beta+c p r_{2}, B=\alpha r_{2}+p \beta+c p r_{2} k-r_{1} r_{2} c-b r_{1} \beta, C=\alpha r_{2} k-r_{1} \beta-r_{1} r_{2} c k$.

Let $\bar{x}(t)=x(t)-x_{*}, \bar{y}(t)=y(t)+y_{*}$. Dropping the bars, system (2) gets the following form:

$$
\left\{\begin{array}{l}
\frac{d x(t)}{d t}=a_{11} x(t)+a_{12} y(t)+b_{11} x\left(t-\tau_{1}\right)+f_{1}, \\
\frac{d y(t)}{d t}=c_{21} x\left(t-\tau_{2}\right)+c_{22} y\left(t-\tau_{2}\right)+f_{2},
\end{array}\right.
$$


where

$$
\begin{aligned}
a_{11}= & \frac{\alpha b x_{*} y_{*}}{\left(1+b x_{*}+c y_{*}\right)^{2}}, \quad a_{12}=-\frac{\alpha x_{*}\left(1+b x_{*}\right)}{\left(1+b x_{*}+c y_{*}\right)^{2}}, \\
b_{11}= & -p x_{*}, \quad c_{21}=\frac{\delta y_{*}^{2}}{\left(x_{*}+k\right)^{2}}, \quad c_{22}=-\frac{\beta y_{*}}{x_{*}+k}, \\
f_{1}= & g_{1} x^{2}(t)+g_{2} x(t) y(t)+g_{3} y^{2}(t)+g_{4} x(t) x\left(t-\tau_{1}\right)+g_{5} x^{2}(t) y(t) \\
& +g_{6} x(t) y^{2}(t)+g_{7} x^{3}(t)+g_{8} y^{3}(t)+\cdots, \\
f_{2}= & h_{1} x^{2}\left(t-\tau_{2}\right)+h_{2} x\left(t-\tau_{2}\right) y(t)+h_{3} x\left(t-\tau_{2}\right) y\left(t-\tau_{2}\right) \\
& +h_{4} x^{2}\left(t-\tau_{2}\right) y(t)+h_{5} x^{2}\left(t-\tau_{2}\right) y\left(t-\tau_{2}\right)+h_{6} x^{3}\left(t-\tau_{2}\right)+\cdots,
\end{aligned}
$$

with

$$
\begin{aligned}
& g_{1}=\frac{b \alpha y_{*}\left(1+c y_{*}\right)}{\left(1+b x_{*}+c y_{*}\right)^{3}}, \quad g_{2}=\frac{\alpha\left(1+b x_{*}+c y_{*}\right)+2 b c \alpha x_{*} y_{*}}{\left(1+b x_{*}+c y_{*}\right)^{3}}, \\
& g_{3}=\frac{c \alpha x_{*}\left(1+b x_{*}\right)}{\left(1+b x_{*}+c y_{*}\right)^{3}}, \quad g_{4}=-p, \quad g_{5}=\frac{b \alpha\left(1+b x_{*}\right)+b c \alpha y_{*}\left(2 b x_{*}-c y_{*}\right)}{\left(1+b x_{*}+c y_{*}\right)^{4}}, \\
& g_{6}=\frac{c \alpha\left(1+c y_{*}\right)+b c \alpha x_{*}\left(2 c y_{*}-b x_{*}\right)}{\left(1+b x_{*}+c y_{*}\right)^{4}}, \quad g_{7}=-\frac{b^{2} \alpha y_{*}\left(1+c y_{*}\right)}{\left(1+b x_{*}+c y_{*}\right)^{4}}, \\
& g_{8}=-\frac{c^{2} \alpha x_{*}\left(1+b x_{*}\right)}{\left(1+b x_{*}+c y_{*}\right)^{4}}, \quad h_{1}=-\frac{\beta y_{*}^{2}}{\left(x_{*}+k\right)^{3}}, \quad h_{2}=\frac{\beta y_{*}}{\left(x_{*}+k\right)^{2}}, \\
& h_{3}=\frac{\beta y_{*}}{\left(x_{*}+k\right)^{2}}, \quad h_{4}=-\frac{\beta y_{*}}{\left(x_{*}+k\right)^{3}}, \quad h_{5}=-\frac{\beta y_{*}}{\left(x_{*}+k\right)^{3}}, \quad h_{6}=\frac{\beta y_{*}^{2}}{\left(x_{*}+k\right)^{4}} .
\end{aligned}
$$

The linearized system of system (3) is

$$
\left\{\begin{array}{l}
\frac{d x}{d t}=a_{11} x(t)+a_{12} y(t)+b_{11} x\left(t-\tau_{1}\right), \\
\frac{d y}{d t}=c_{21} x\left(t-\tau_{2}\right)+c_{22} y\left(t-\tau_{2}\right) .
\end{array}\right.
$$

The characteristic equation of system (15) is

$$
\lambda^{2}+A_{1} \lambda+B_{1} \lambda e^{-\lambda \tau_{1}}+\left(C_{1} \lambda+C_{0}\right) e^{-\lambda \tau_{2}}+D_{0} e^{-\lambda\left(\tau_{1}+\tau_{2}\right)}=0
$$

where

$$
A_{1}=-a_{11}, \quad B_{1}=-b_{11}, \quad C_{0}=a_{11} c_{22}-a_{12} c_{21}, \quad C_{1}=-c_{22}, \quad D_{0}=b_{11} c_{22} .
$$

Case 1. $\tau_{1}=\tau_{2}=0$.

When $\tau_{1}=\tau_{2}=0$, Eq. (16) becomes

$$
\lambda^{2}+A_{11} \lambda+A_{10}=0
$$

where

$$
A_{10}=C_{0}+D_{0}, \quad A_{11}=A_{1}+B_{1}+C_{1} .
$$


It is easy to obtain $A_{11}=r_{1}+r_{2}+\frac{\alpha y_{*}\left(1+c y_{*}\right)}{\left(1+b x_{*}+c y_{*}\right)^{2}}>0, A_{10}=\left(a_{11}+b_{11}\right) c_{22}-a_{12} c_{21}$. From the expressions of $a_{11}$ and $b_{11}$, we get $a_{11}+b_{11}=-r_{1}-\frac{\alpha y_{*}\left(1+c y_{*}\right)}{\left(1+b x_{*}+c y_{*}\right)^{2}}<0$. Then we can get $A_{10}>0$. Therefore, if the condition $\left(\mathrm{H}_{1}\right)$ holds, then the positive equilibrium of system (2) without delay is locally asymptotically stable.

Case 2. $\tau_{1}>0, \tau_{2}=0$.

When $\tau_{1}>0, \tau_{2}=0$, Eq. (16) becomes

$$
\lambda^{2}+A_{21} \lambda+A_{20}+\left(B_{21} \lambda+B_{20}\right) e^{-\lambda \tau_{1}}=0,
$$

where

$$
A_{21}=A_{1}+C_{1}, \quad A_{20}=C_{0}, \quad B_{21}=B_{1}, \quad B_{20}=D_{0} .
$$

Let $\lambda=i \omega_{1}\left(\omega_{1}>0\right)$ be the root of Eq. (18). Then we have

$$
\left\{\begin{array}{l}
B_{21} \omega_{1} \cos \tau_{1} \omega_{1}-B_{20} \omega_{1} \sin \tau_{1} \omega_{1}=-A_{21} \omega_{1} \\
B_{21} \omega_{1} \sin \tau_{1} \omega_{1}+B_{20} \omega_{1} \cos \tau_{1} \omega_{1}=\omega_{1}^{2}-A_{20}
\end{array}\right.
$$

from which one can obtain

$$
\omega_{1}^{4}+\left(A_{21}^{2}-B_{21}^{2}-2 A_{20}\right) \omega_{1}^{2}+A_{20}^{2}-B_{20}^{2}=0 .
$$

Since $C_{0}+D_{0}>0$, we can conclude that if we have the condition $\left(\mathrm{H}_{2}\right): C_{0}-D_{0}<0$, then $A_{20}<B_{20}$, and further Eq. (19) has a unique positive root

$$
\omega_{10}=\sqrt{\frac{-\left(A_{21}^{2}-B_{21}^{2}-2 A_{20}\right)+\sqrt{\left(A_{21}^{2}-B_{21}^{2}-2 A_{20}\right)^{2}-4\left(A_{20}^{2}-B_{20}^{2}\right)}}{2}},
$$

and the corresponding critical value of the delay is

$$
\tau_{1 k}=\frac{1}{\omega_{10}} \arccos \frac{\left(B_{20}-A_{21} B_{21}\right) \omega_{10}^{2}-A_{20} B_{20}}{B_{21}^{2} \omega_{10}^{2}+B_{20}^{2}}+\frac{2 k \pi}{\omega_{10}}, \quad k=0,1,2, \ldots
$$

Define

$$
\tau_{10}=\frac{1}{\omega_{10}} \arccos \frac{\left(B_{20}-A_{21} B_{21}\right) \omega_{10}^{2}-A_{20} B_{20}}{B_{21}^{2} \omega_{10}^{2}+B_{20}^{2}} .
$$

When $\tau_{1}=\tau_{10}$, then Eq. (18) has a pair of purely imaginary roots $\pm i \omega_{10}$. Differentiating the two sides of Eq. (18), one can obtain

$$
\left[\frac{d \lambda}{d \tau_{1}}\right]^{-1}=-\frac{2 \lambda+A_{21}}{\lambda\left(\lambda^{2}+A_{21} \lambda+A_{20}\right)}+\frac{B_{21}}{\lambda\left(B_{21} \lambda+B_{20}\right)}-\frac{\tau_{1}}{\lambda} .
$$

Thus,

$$
\operatorname{Re}\left[\frac{d \lambda}{d \tau_{1}}\right]_{\tau_{1}=\tau_{10}}^{-1}=\frac{\sqrt{\left(A_{21}^{2}-B_{21}^{2}-2 A_{20}\right)^{2}-4\left(A_{20}^{2}-B_{20}^{2}\right)}}{B_{21}^{2} \omega_{10}^{2}+B_{20}^{2}}>0 .
$$


Based on the analysis above and according to the Hopf bifurcation theorem in [21], we have the following results.

Theorem 1 Suppose that the conditions $\left(\mathrm{H}_{1}\right)$ and $\left(\mathrm{H}_{2}\right)$ hold. The positive equilibrium $E_{*}\left(x_{*}, y_{*}\right)$ of system (2) is asymptotically stable for $\tau_{1} \in\left[0, \tau_{10}\right)$. System (2) undergoes a Hopf bifurcation at the positive equilibrium $E_{*}\left(x_{*}, y_{*}\right)$ of system (2) when $\tau_{1}=\tau_{10}$ and a family of periodic solutions bifurcate from the positive equilibrium $E_{*}\left(x_{*}, y_{*}\right)$ of system (2).

Case 3. $\tau_{1}=0, \tau_{2}>0$.

Substitute $\tau_{1}=0$ into Eq. (16), then Eq. (16) becomes

$$
\lambda^{2}+A_{31} \lambda+\left(B_{31} \lambda+B_{30}\right) e^{-\lambda \tau_{2}}=0,
$$

where

$$
\begin{aligned}
& A_{31}=A_{1}+B_{1}, \quad B_{31}=C_{1}, \quad B_{30}=C_{0}+D_{0}, \\
& \left\{\begin{array}{l}
B_{31} \omega_{2} \sin \omega_{2} \tau_{2}+B_{30} \cos \omega_{2} \tau_{2}=\omega_{2}^{2}, \\
B_{31} \omega_{2} \cos \omega_{2} \tau_{2}-B_{30} \sin \omega_{2} \tau_{2}=-A_{31} \omega_{2},
\end{array}\right.
\end{aligned}
$$

from which it follows that

$$
\omega_{2}^{4}+\left(A_{31}^{2}-B_{31}^{2}\right) \omega_{2}^{4}-B_{30}^{2}=0 .
$$

Obviously, $B_{30}^{2}>0$. Thus, we can conclude that Eq. (21) has a unique positive root

$$
\omega_{20}=\sqrt{\frac{-\left(A_{31}^{2}-B_{31}^{2}\right)+\sqrt{\left(A_{31}^{2}-B_{31}^{2}\right)^{2}+4 B_{30}^{2}}}{2}},
$$

and the corresponding critical value of the delay is

$$
\tau_{2 k}=\frac{1}{\omega_{20}} \arccos \frac{\left(B_{30}-A_{31} B_{31}\right) \omega_{20}^{2}}{B_{31}^{2} \omega_{20}^{2}+B_{30}^{2}}+\frac{2 k \pi}{\omega_{20}}, \quad k=0,1,2, \ldots
$$

Define

$$
\tau_{20}=\frac{1}{\omega_{20}} \arccos \frac{\left(B_{30}-A_{31} B_{31}\right) \omega_{20}^{2}}{B_{31}^{2} \omega_{20}^{2}+B_{30}^{2}} .
$$

When $\tau_{2}=\tau_{20}$, then Eq. (20) has a pair of purely imaginary roots $\pm i \omega_{20}$ and similar to Case 2, we can obtain

$$
\operatorname{Re}\left[\frac{d \lambda}{d \tau_{2}}\right]_{\tau_{2}=\tau_{20}}^{-1}=\frac{\sqrt{\left(A_{31}-B_{31}\right)^{2}+4 B_{30}^{2}}}{B_{31}^{2} \omega_{20}^{2}+B_{30}^{2}}>0 .
$$

Based on the analysis above and according to the Hopf bifurcation theorem in [21], we have the following results. 
Theorem 2 Suppose that the condition $\left(\mathrm{H}_{1}\right)$ holds. The positive equilibrium $E_{*}\left(x_{*}, y_{*}\right)$ of system (2) is asymptotically stable for $\tau_{2} \in\left[0, \tau_{20}\right)$. System (2) undergoes a Hopf bifurcation at the positive equilibrium $E_{*}\left(x_{*}, y_{*}\right)$ of system (2) when $\tau_{2}=\tau_{20}$ and a family of periodic solutions bifurcate from the positive equilibrium $E_{*}\left(x_{*}, y_{*}\right)$ of system (2).

Case 4. $\tau_{1}=\tau_{2}=\tau>0$.

When $\tau_{1}=\tau_{2}=\tau$, then Eq. (16) becomes

$$
\lambda^{2}+A_{41} \lambda+\left(B_{41} \lambda+B_{40}\right) e^{-\lambda \tau}+C_{40} e^{-2 \lambda \tau}=0,
$$

where

$$
A_{41}=A_{1}, \quad B_{41}=B_{1}+C_{1}, \quad B_{40}=C_{0}, \quad C_{40}=D_{0} .
$$

Multiplying by $e^{\lambda \tau}$, Eq. (22) becomes

$$
B_{41} \lambda+B_{40}+\left(\lambda^{2}+A_{41} \lambda\right) e^{\lambda \tau}+C_{40} e^{-\lambda \tau}=0 .
$$

Let $\lambda=i \omega(\omega>0)$ be the root of Eq. (23). Then

$$
\left\{\begin{array}{l}
\left(\omega^{2}-C_{40}\right) \cos \tau \omega+A_{41} \omega \sin \tau \omega=B_{40} \\
\left(\omega^{2}-C_{40}\right) \sin \tau \omega-A_{41} \omega \cos \tau \omega=B_{41} \omega
\end{array}\right.
$$

It follows that

$$
\cos \tau \omega=\frac{\left(B_{40}-A_{41} B_{41}\right) \omega^{2}+B_{40} C_{40}}{\omega^{4}+A_{41}^{2} \omega^{2}-C_{40}^{2}}, \quad \cos \tau \omega=\frac{\left(B_{40}-A_{41} B_{41}\right) \omega^{2}+B_{40} C_{40}}{\omega^{4}+A_{41}^{2} \omega^{2}-C_{40}^{2}} .
$$

Then we have

$$
\omega^{8}+e_{43} \omega^{6}+e_{42} \omega^{4}+e_{41} \omega^{2}+e_{40}=0
$$

where

$$
\begin{aligned}
& e_{40}=C_{40}^{2}\left(C_{40}^{2}-B_{40}^{2}\right), \quad e_{43}=2 A_{41}^{2}-B_{41}^{2}, \\
& e_{41}=2 B_{40} C_{40}\left(A_{41} B_{41}-B_{40}\right)-\left(A_{41} B_{40}-B_{41} C_{40}\right)^{2}-2 A_{41}^{2} C_{40}^{2}, \\
& e_{42}=A_{41}^{4}-2 C_{40}^{2}-2 B_{41}\left(A_{41} B_{40}-B_{41} C_{40}\right)-\left(B_{40}-A_{41} B_{41}\right)^{2} .
\end{aligned}
$$

Let $\omega^{2}=v$, then Eq. (24) becomes

$$
v^{4}+e_{43} v^{3}+e_{42} v^{2}+e_{41} v+e_{40}=0
$$

The discussion of the roots of Eq. (28) is similar to that in [22]. Denote

$$
f(v)=v^{4}+e_{43} v^{3}+e_{42} v^{2}+e_{41} v+e_{40} .
$$


From Eq. (29), we have

$$
f^{\prime}(v)=4 v^{3}+3 e_{43} v^{2}+2 e_{42} v+e_{41} .
$$

Set

$$
4 v^{3}+3 e_{43} v^{2}+2 e_{42} v+e_{41}=0 .
$$

Let $z=\frac{3 e_{43}}{4}+v$. Then Eq. (29) becomes

$$
z^{3}+\alpha_{41} z+\alpha_{40}=0,
$$

where

$$
\alpha_{41}=\frac{1}{2} e_{42}-\frac{3}{16} e_{43}^{2}, \quad \alpha_{40}=\frac{1}{32} e_{43}^{3}-\frac{1}{8} e_{42} e_{43}+e_{41} .
$$

Define

$$
\begin{aligned}
& \gamma_{41}=\left(\frac{\alpha_{40}}{2}\right)^{2}+\left(\frac{\alpha_{41}}{3}\right)^{3}, \quad \gamma_{42}=\frac{-1+\sqrt{3} i}{2} \\
& z_{1}=\sqrt[3]{-\frac{\alpha_{40}}{2}+\sqrt{\gamma_{41}}}+\sqrt[3]{-\frac{\alpha_{40}}{2}-\sqrt{\gamma_{41}}} \\
& z_{2}=\sqrt[3]{-\frac{\alpha_{40}}{2}+\sqrt{\gamma_{41}} \gamma_{42}}+\sqrt[3]{-\frac{\alpha_{40}}{2}-\sqrt{\gamma_{41}} \gamma_{42}^{2}} \\
& z_{3}=\sqrt[3]{-\frac{\alpha_{40}}{2}+\sqrt{\gamma_{41}} \gamma_{42}^{2}}+\sqrt[3]{-\frac{\alpha_{40}}{2}-\sqrt{\gamma_{41}} \gamma_{42}} \\
& v_{i}=z_{i}-\frac{3 e_{43}}{4}, \quad i=1,2,3
\end{aligned}
$$

Based on the conditions $\left(\mathrm{H}_{1}\right)$ and $\left(\mathrm{H}_{2}\right)$, we can conclude that $e_{40}>0$. Then we have the following results according to Lemma 2.2 in [22].

Lemma 1 For Eq. (29),

(i) if $\gamma_{41} \geq 0$, then Eq. (29) has positive root if and only if $v_{1}>0$ and $f\left(v_{1}\right)<0$;

(ii) if $\gamma_{41}<0$, then Eq. (29) has positive root if and only if there exists at least one $v_{*} \in\left\{v_{1}, v_{2}, v_{3}\right\}$, such that $v_{*}>0$ and $f\left(v_{*}\right) \leq 0$.

In what follows, we assume that $\left(\mathrm{H}_{41}\right)$ : the coefficients satisfy one of the following conditions in $\alpha^{\prime}-\beta^{\prime}$ : $\left(\alpha^{\prime}\right) \gamma_{41} \geq 0, v_{1}>0$, and $f\left(v_{1}\right)<0 ;\left(\beta^{\prime}\right) \gamma_{41}<0$ and there exists at least one $v_{*} \in\left\{v_{1}, v_{2}, v_{3}\right\}$, such that $v_{*}>0$ and $f\left(v_{*}\right) \leq 0$.

If the condition $\left(\mathrm{H}_{41}\right)$ holds, Eq. (28) has at least one positive root $v_{0}$. Thus, Eq. (24) has at least one positive root $\omega_{0}=\sqrt{v_{0}}$ and the corresponding critical value of the delay is

$$
\tau_{k}=\frac{1}{\omega_{0}} \arccos \frac{\left(B_{40}-A_{41} B_{41}\right) \omega_{0}^{2}+B_{40} C_{40}}{\omega_{0}^{4}+A_{41}^{2} \omega_{0}^{2}-C_{40}^{2}}+\frac{2 j \pi}{\omega_{0}}, \quad k=0,1,2, \ldots
$$

Let

$$
\tau_{0}=\frac{1}{\omega_{0}} \arccos \frac{\left(B_{40}-A_{41} B_{41}\right) \omega_{0}^{2}+B_{40} C_{40}}{\omega_{0}^{4}+A_{41}^{2} \omega_{0}^{2}-C_{40}^{2}} .
$$


When $\tau=\tau_{0}$, Eq. (23) has a pair of purely imaginary roots $\pm i \omega_{0}$. Differentiating both sides of Eq. (23) with respect to $\tau$, we get

$$
\left[\frac{d \lambda}{d \tau}\right]^{-1}=\frac{\left(2 \lambda+A_{41}\right) e^{\lambda \tau}+B_{41}}{C_{40} \lambda e^{\lambda \tau}-\left(\lambda^{3}+A_{41} \lambda^{2}\right) e^{-\lambda \tau}}-\frac{\tau}{\lambda}
$$

Thus,

$$
\operatorname{Re}\left[\frac{d \lambda}{d \tau}\right]_{\tau=\tau_{0}}^{-1}=\frac{P_{4 R} Q_{4 R}+P_{4 I} Q_{4 I}}{Q_{4 R}^{2}+Q_{4 I}^{2}}
$$

where

$$
\begin{aligned}
& P_{4 R}=A_{41} \cos \tau_{0} \omega_{0}-2 \omega_{0} \sin \tau_{0} \omega_{0}+B_{41}, \\
& P_{4 I}=A_{41} \sin \tau_{0} \omega_{0}+2 \omega_{0} \cos \tau_{0} \omega_{0}, \\
& Q_{4 R}=A_{41} \omega_{0}^{2} \cos \tau_{0} \omega_{0}+\left(C_{40} \omega_{0}-\omega_{0}^{3}\right) \sin \tau_{0} \omega_{0}, \\
& Q_{4 I}=A_{41} \omega_{0}^{2} \sin \tau_{0} \omega_{0}+\left(C_{40} \omega_{0}+\omega_{0}^{3}\right) \cos \tau_{0} \omega_{0} .
\end{aligned}
$$

Obviously, if the condition $\left(\mathrm{H}_{42}\right): P_{4 R} Q_{4 R}+P_{4 I} Q_{4 I} \neq 0$ holds, the transversality condition is satisfied. Based on the discussion above and according to the Hopf bifurcation theorem in [21] we have the following results.

Theorem 3 Suppose that the conditions $\left(\mathrm{H}_{1}\right),\left(\mathrm{H}_{2}\right),\left(\mathrm{H}_{41}\right)$, and $\left(\mathrm{H}_{42}\right)$ hold. The positive equilibrium $E_{*}\left(x_{*}, y_{*}\right)$ of system (2) is asymptotically stable for $\tau \in\left[0, \tau_{0}\right)$. System (2) undergoes a Hopf bifurcation at the positive equilibrium $E_{*}\left(x_{*}, y_{*}\right)$ of system (2) when $\tau=\tau_{0}$ and a family of periodic solutions bifurcate from the positive equilibrium $E_{*}\left(x_{*}, y_{*}\right)$ of system (2).

Case 5. $\tau_{1}>0, \tau_{2} \in\left(0, \tau_{20}\right)$.

We consider Eq. (16) with $\tau_{2}$ in its stable interval and $\tau_{1}$ is considered as the bifurcation parameter. Let $\lambda=i \omega_{1}^{\prime}\left(\omega_{1}^{\prime}>0\right)$ be the root of Eq. (16). Then

$$
\left\{\begin{array}{l}
M_{51} \sin \tau_{1} \omega_{1}^{\prime}+M_{52} \cos \tau_{1} \omega_{1}^{\prime}=N_{51}, \\
M_{51} \cos \tau_{1} \omega_{1}^{\prime}-M_{52} \sin \tau_{1} \omega_{1}^{\prime}=N_{52},
\end{array}\right.
$$

with

$$
\begin{aligned}
& M_{51}=B_{1} \omega_{1}^{\prime}-D_{0} \sin \tau_{2} \omega_{1}^{\prime}, \quad M_{52}=D_{0} \cos \tau_{2} \omega_{1}^{\prime}, \\
& N_{51}=\left(\omega_{1}^{\prime}\right)^{2}-C_{1} \omega_{1}^{\prime} \sin \tau_{2} \omega_{1}^{\prime}-C_{0} \cos \tau_{2} \omega_{1}^{\prime}, \\
& N_{52}=-A_{1} \omega_{1}^{\prime}-C_{1} \omega_{1}^{\prime} \cos \tau_{2} \omega_{1}^{\prime}+C_{0} \sin \tau_{2} \omega_{1}^{\prime} .
\end{aligned}
$$

Then one can obtain

$$
e_{50}\left(\omega_{1}^{\prime}\right)+e_{51}\left(\omega_{1}^{\prime}\right) \cos \tau_{2} \omega_{1}^{\prime}+e_{52} \sin \tau_{2} \omega_{1}^{\prime}=0
$$


where

$$
\begin{aligned}
& e_{50}\left(\omega_{1}^{\prime}\right)=\left(\omega_{1}^{\prime}\right)^{4}+\left(A_{1}^{2}-B_{1}^{2}+C_{1}^{2}\right)\left(\omega_{1}^{\prime}\right)^{2}+C_{0}^{2}-D_{0}^{2}, \\
& e_{51}\left(\omega_{1}^{\prime}\right)=2\left(A_{1} C_{1}-C_{0}\right)\left(\omega_{1}^{\prime}\right)^{2}, \\
& e_{52}\left(\omega_{1}^{\prime}\right)=-2 C_{1}\left(\omega_{1}^{\prime}\right)^{3}+2\left(B_{1} D_{0}-A_{1} C_{0}\right) \omega_{1}^{\prime} .
\end{aligned}
$$

In order to give the main results in this paper, we make the following assumption. $\left(\mathrm{H}_{51}\right)$ : Eq. (44) has at least finite positive roots. If the condition $\left(\mathrm{H}_{51}\right)$ holds, we denote the roots of Eq. (44) as $\omega_{11}^{\prime}, \omega_{12}^{\prime}, \ldots, \omega_{1 k}^{\prime}$. For every fixed $\omega_{1 i}^{\prime}(i=1,2, \ldots, k)$, the corresponding critical value of the delay is

$$
\tau_{1 i}^{(j) \prime}=\frac{1}{\omega_{1 i}^{\prime}} \arccos \left\{\frac{M_{51} N_{52}+M_{52} N_{51}}{M_{51}^{2}+M_{52}^{2}}\right\}_{\omega_{1}^{\prime}=\omega_{1 i}^{\prime}}+\frac{2 j \pi}{\omega_{1 i}^{\prime}}, \quad i=1,2, \ldots, k, j=0,1,2, \ldots
$$

Let $\tau_{10}^{*}=\min \left\{\tau_{1 i}^{(j) \prime} \mid i=1,2, \ldots, k, j=0,1,2, \ldots\right\}$. When $\tau_{1}=\tau_{10}^{*}$, Eq. (16) has a pair of purely imaginary roots $\pm i \omega_{1}^{*}$ for $\tau_{2} \in\left(0, \tau_{20}\right)$. Differentiating Eq. (16) with respect to $\tau_{1}$, one can obtain

$$
\left[\frac{d \lambda}{d \tau_{1}}\right]^{-1}=\frac{2 \lambda+A_{1}+B_{1} e^{-\lambda \tau_{1}}+\left(C_{1}-\tau_{2} C_{0}-\tau_{2} C_{1}\right) e^{-\lambda \tau_{2}}-\tau_{2} D_{0} e^{-\lambda\left(\tau_{1}+\tau_{2}\right)}}{B_{1} \lambda^{2} e^{-\lambda \tau_{1}}+D_{0} \lambda e^{-\lambda\left(\tau_{1}+\tau_{2}\right)}} .
$$

Thus,

$$
\left[\frac{d \lambda}{d \tau_{1}}\right]_{\tau_{1}=\tau_{10}^{*}}^{-1}=\frac{P_{5 R} Q_{5 R}+P_{5 I} Q_{5 I}}{Q_{5 R}^{2}+Q_{5 I}^{2}}
$$

where

$$
\begin{aligned}
P_{5 R}= & A_{1}+\left(C_{1}-\tau_{2} C_{1}-\tau_{2} C_{0}\right) \cos \tau_{2} \omega_{1}^{*} \\
& +\left(B_{1}+\tau_{2} D_{0} \cos \tau_{2} \omega_{1}^{*}\right) \cos \tau_{10}^{*} \omega_{1}^{*}-\tau_{2} D_{0} \sin \tau_{2} \omega_{1}^{*} \sin \tau_{10}^{*} \omega_{1}^{*}, \\
P_{5 I}= & 2 \omega_{1}^{*}-\left(C_{1}-\tau_{2} C_{1}-\tau_{2} C_{0}\right) \sin \tau_{2} \omega_{1}^{*} \\
& -\left(B_{1}+\tau_{2} D_{0} \cos \tau_{2} \omega_{1}^{*}\right)-\tau_{2} D_{0} \sin \tau_{2} \omega_{1}^{*} \cos \tau_{10}^{*} \omega_{1}^{*}, \\
Q_{5 R}= & \left(D_{0} \omega_{1}^{*} \sin \tau_{2} \omega_{1}^{*}-B_{1}\left(\omega_{1}^{*}\right)^{2}\right) \cos \tau_{10}^{*} \omega_{1}^{*}+D_{0} \omega_{1}^{*} \cos \tau_{2} \omega_{1}^{*} \sin \tau_{10}^{*} \omega_{1}^{*}, \\
Q_{5 I}= & \left(B_{1}\left(\omega_{1}^{*}\right)^{2}-D_{0} \omega_{1}^{*} \sin \tau_{2} \omega_{1}^{*}\right) \sin \tau_{10}^{*} \omega_{1}^{*}+D_{0} \omega_{1}^{*} \cos \tau_{2} \omega_{1}^{*} \cos \tau_{10}^{*} \omega_{1}^{*} .
\end{aligned}
$$

Obviously, if the condition $\left(\mathrm{H}_{52}\right): P_{5 R} Q_{5 R}+P_{5 I} Q_{5 I} \neq 0$ holds, then $\operatorname{Re}\left[\frac{d \lambda}{d \tau_{1}}\right]_{\tau_{1}=\tau_{10}^{*}}^{-1} \neq 0$. Thus, according to the Hopf bifurcation theorem in [21] we have the following result.

Theorem 4 Suppose that the conditions $\left(\mathrm{H}_{1}\right),\left(\mathrm{H}_{51}\right)$, and $\left(\mathrm{H}_{52}\right)$ hold and $\tau_{2} \in\left(0, \tau_{20}\right)$. The positive equilibrium $E_{*}\left(x_{*}, y_{*}\right)$ of system (2) is asymptotically stable for $\tau_{1} \in\left[0, \tau_{10}^{*}\right)$. System (2) undergoes a Hopf bifurcation at the positive equilibrium $E_{*}\left(x_{*}, y_{*}\right)$ of system (2) when $\tau_{1}=\tau_{10}^{*}$ and a family of periodic solutions bifurcate from the positive equilibrium $E_{*}\left(x_{*}, y_{*}\right)$ of system (2). 
Case 6. $\tau_{2}>0, \tau_{1} \in\left(0, \tau_{10}\right)$

We consider Eq. (16) with $\tau_{1}$ in its stable interval and $\tau_{2}$ is considered as the bifurcation parameter. Let $\lambda=i \omega_{2}^{\prime}\left(\omega_{2}^{\prime}>0\right)$ be the root of Eq. (16). Then

$$
\left\{\begin{array}{l}
M_{61} \sin \tau_{2} \omega_{2}^{\prime}+M_{62} \cos \tau_{2} \omega_{2}^{\prime}=N_{61}, \\
M_{61} \cos \tau_{2} \omega_{2}^{\prime}-M_{62} \sin \tau_{2} \omega_{2}^{\prime}=N_{62}
\end{array}\right.
$$

with

$$
\begin{aligned}
& M_{61}=C_{1} \omega_{2}^{\prime}-D_{0} \sin \tau_{1} \omega_{2}^{\prime}, \quad M_{62}=C_{0}+D_{0} \cos \tau_{1} \omega_{2}^{\prime}, \\
& N_{61}=\left(\omega_{2}^{\prime}\right)^{2}-B_{1} \omega_{2}^{\prime} \sin \tau_{1} \omega_{2}^{\prime}, \quad N_{62}=-A_{1} \omega_{2}^{\prime}-B_{1} \omega_{2}^{\prime} \cos \tau_{1} \omega_{2}^{\prime} .
\end{aligned}
$$

It follows that

$$
e_{60}\left(\omega_{2}^{\prime}\right)+e_{61}\left(\omega_{2}^{\prime}\right) \cos \tau_{1} \omega_{2}^{\prime}+e_{62} \sin \tau_{1} \omega_{2}^{\prime}=0,
$$

where

$$
\begin{aligned}
& e_{60}\left(\omega_{2}^{\prime}\right)=\left(\omega_{2}^{\prime}\right)^{4}+\left(A_{1}^{2}+B_{1}^{2}-C_{1}^{2}\right)\left(\omega_{2}^{\prime}\right)^{2}-C_{0}^{2}+D_{0}^{2}, \\
& e_{51}\left(\omega_{2}^{\prime}\right)=2 A_{1} B_{1}\left(\omega_{2}^{\prime}\right)^{2}-2 C_{0} D_{0}, \\
& e_{52}\left(\omega_{2}^{\prime}\right)=-2 B_{1}\left(\omega_{2}^{\prime}\right)^{3}+2 C_{1} D_{0} \omega_{2}^{\prime} .
\end{aligned}
$$

Similar to Case 5, we make the following assumption. $\left(\mathrm{H}_{61}\right)$ : Eq. (56) has at least finite positive roots. We denote the roots of Eq. (56) as $\omega_{21}^{\prime}, \omega_{22}^{\prime}, \ldots, \omega_{2 k}^{\prime}$. For every fixed $\omega_{2 i}^{\prime}(i=$ $1,2, \ldots, k)$, the corresponding critical value of the delay is

$$
\tau_{2 i}^{(j) \prime}=\frac{1}{\omega_{2 i}^{\prime}} \arccos \left\{\frac{M_{61} N_{62}+M_{62} N_{61}}{M_{61}^{2}+M_{62}^{2}}\right\}_{\omega_{2}^{\prime}=\omega_{2 i}^{\prime}}+\frac{2 j \pi}{\omega_{2 i}^{\prime}}, \quad i=1,2, \ldots, k, j=0,1,2, \ldots
$$

Let $\tau_{20}^{*}=\min \left\{\tau_{2 i}^{(j) \prime} \mid i=1,2, \ldots, k, j=0,1,2, \ldots\right\}$. When $\tau_{2}=\tau_{20}^{*}$, Eq. (16) has a pair of purely imaginary roots $\pm i \omega_{2}^{*}$ for $\tau_{1} \in\left(0, \tau_{10}\right)$. Differentiating Eq. (16) with respect to $\tau_{2}$, one can obtain

$$
\left[\frac{d \lambda}{d \tau_{2}}\right]^{-1}=\frac{2 \lambda+A_{1}+\left(B_{1}-\tau_{1} B_{1} \lambda\right) e^{-\lambda \tau_{1}}+C_{1} e^{-\lambda \tau_{2}}-\tau_{1} D_{0} e^{-\lambda\left(\tau_{1}+\tau_{2}\right)}}{\left(C_{1} \lambda^{2}+C_{0} \lambda\right) e^{-\lambda \tau_{2}}+D_{0} \lambda e^{-\lambda\left(\tau_{1}+\tau_{2}\right)}}-\frac{\tau_{2}}{\lambda} .
$$

Thus,

$$
\left[\frac{d \lambda}{d \tau_{2}}\right]_{\tau_{2}=\tau_{20}^{*}}^{-1}=\frac{P_{6 R} Q_{6 R}+P_{6 I} Q_{6 I}}{Q_{6 R}^{2}+Q_{6 I}^{2}}
$$

where

$$
\begin{aligned}
P_{6 R}= & \left(C_{1}-\tau_{1} D_{0} \cos \tau_{1} \omega_{2}^{*}\right) \cos \tau_{20}^{*} \omega_{2}^{*}+\tau_{1} D_{0} \sin \tau_{1} \omega_{2}^{*} \sin \tau_{20}^{*} \omega_{2}^{*} \\
& +A_{1}+B_{1} \cos \tau_{1} \omega_{2}^{*}+\tau_{1} B_{1} \omega_{2}^{*} \sin \tau_{1} \omega_{2}^{*}, \\
P_{6 I}= & \left(\tau_{1} D_{0} \cos \tau_{1} \omega_{2}^{*}-C_{1}\right) \sin \tau_{20}^{*} \omega_{2}^{*}+\tau_{1} D_{0} \sin \tau_{1} \omega_{2}^{*} \cos \tau_{20}^{*} \omega_{2}^{*}
\end{aligned}
$$




$$
\begin{aligned}
& +2 \omega_{2}^{*}-B_{1} \sin \tau_{1} \omega_{2}^{*}-\tau_{1} B_{1} \omega_{2}^{*} \cos \tau_{1} \omega_{2}^{*}, \\
Q_{6 R}= & \left(C_{0} \omega_{2}^{*}+D_{0} \omega_{2}^{*} \cos \tau_{1} \omega_{2}^{*}\right) \sin \tau_{20}^{*} \omega_{2}^{*}+\left(D_{0} \omega_{2}^{*} \sin \tau_{1} \omega_{2}^{*}-C_{1}\left(\omega_{2}^{*}\right)^{2}\right) \cos \tau_{20}^{*} \omega_{2}^{*}, \\
Q_{6 I}= & \left(C_{0} \omega_{2}^{*}+D_{0} \omega_{2}^{*} \cos \tau_{1} \omega_{2}^{*}\right) \cos \tau_{20}^{*} \omega_{2}^{*}-\left(D_{0} \omega_{2}^{*} \sin \tau_{1} \omega_{2}^{*}-C_{1}\left(\omega_{2}^{*}\right)^{2}\right) \sin \tau_{20}^{*} \omega_{2}^{*} .
\end{aligned}
$$

Similar as in Case 5, we can conclude that if the condition $\left(\mathrm{H}_{62}\right): P_{6 R} Q_{6 R}+P_{6 I} Q_{6 I} \neq 0$ holds, then $\operatorname{Re}\left[\frac{d \lambda}{d \tau_{2}}\right]_{\tau_{2}=\tau_{20}^{*}}^{-1} \neq 0$. Thus, according to the Hopf bifurcation theorem in [21] we have the following results.

Theorem 5 Suppose that the conditions $\left(\mathrm{H}_{1}\right),\left(\mathrm{H}_{61}\right)$, and $\left(\mathrm{H}_{62}\right)$ hold and $\tau_{1} \in\left(0, \tau_{10}\right)$. The positive equilibrium $E_{*}\left(x_{*}, y_{*}\right)$ of system (2) is asymptotically stable for $\tau_{2} \in\left[0, \tau_{20}^{*}\right)$. System (2) undergoes a Hopf bifurcation at the positive equilibrium $E_{*}\left(x_{*}, y_{*}\right)$ of system (2) when $\tau_{2}=\tau_{20}^{*}$ and a family of periodic solutions bifurcate from the positive equilibrium $E_{*}\left(x_{*}, y_{*}\right)$ of system (2).

\section{Direction and stability of bifurcated periodic solutions}

In this section, we investigate the direction of the Hopf bifurcation and the stability of bifurcated periodic solutions of system (2) with respect to $\tau_{2}$ for $\tau_{1} \in\left(0, \tau_{10}\right)$. Let $\tau_{2}=\tau_{20}^{*}+\mu$, $\mu \in R$, so that the Hopf bifurcation occurs at $\mu=0$. Without loss of generality, we assume that $\tau_{1 *}<\tau_{20}^{*}$, where $\tau_{1 *} \in\left(0, \tau_{10}\right)$.

Let $u_{1}(t)=x(t)-x_{*}, u_{2}(t)=y(t)-y_{*}$, and rescale the time delay $t \rightarrow\left(t / \tau_{2}\right)$, then system (2) becomes

$$
\dot{u}(t)=L_{\mu} u_{t}+F\left(\mu, u_{t}\right)
$$

where $u(t)=\left(u_{1}(t), u_{2}(t)\right)^{T} \in R^{2}$ and $L_{\mu}: C \rightarrow R^{2}, F: R \times C \rightarrow R^{2}$ are given, respectively, by

$$
L_{\mu} \phi=\left(\tau_{20}^{*}+\mu\right)\left(A^{\prime} \phi(0)+B^{\prime} \phi\left(-\frac{\tau_{1 *}}{\tau_{20}^{\prime}}\right)+C^{\prime} \phi(-1)\right)
$$

and

$$
F(\mu, \phi)=\left(\tau_{20}^{*}+\mu\right)\left(F_{1}, F_{2}\right)^{T},
$$

with $\phi(\theta)=\left(\phi_{1}(\theta), \phi_{2}(\theta)\right)^{T} \in C\left([-1,0], R^{2}\right)$,

$$
A^{\prime}=\left(\begin{array}{cc}
a_{11} & a_{12} \\
0 & 0
\end{array}\right), \quad B^{\prime}=\left(\begin{array}{cc}
b_{11} & 0 \\
0 & 0
\end{array}\right), \quad C^{\prime}=\left(\begin{array}{cc}
0 & 0 \\
c_{21} & c_{22}
\end{array}\right)
$$

and

$$
\begin{aligned}
F_{1}= & g_{1} \phi_{1}^{2}(0)+g_{2} \phi_{1}(0) \phi_{2}(0)+g_{3} \phi_{2}^{2}(0)+g_{4} \phi_{1}(0) \phi_{1}\left(-\frac{\tau_{1 *}}{\tau_{20}^{*}}\right)+g_{5} \phi_{1}^{2}(0) \phi_{2}(0) \\
& +g_{6} \phi_{1}(0) \phi_{2}^{2}(0)+g_{7} \phi_{1}^{3}(0)+g_{8} \phi_{2}^{3}(0)+\cdots \\
F_{2}= & h_{1} \phi_{1}^{2}(-1)+h_{2} \phi_{1}(-1) \phi_{2}(0)+h_{3} \phi_{1}(-1) \phi_{2}(-1)+h_{4} \phi_{1}^{2}(-1) \phi_{2}(0) \\
& +h_{5} \phi_{1}^{2}(-1) \phi_{2}(-1)+h_{6} \phi_{1}^{3}(-1)+\cdots
\end{aligned}
$$


Using the Riesz representation theorem, there exists a $2 \times 2$ matrix function $\eta(\theta, \mu)$, $\theta \in[-1,0] \rightarrow R^{2 \times 2}$ whose elements are of bounded variation, such that

$$
L_{\mu} \phi=\int_{-1}^{0} d \eta(\theta, \mu) \phi(\theta), \quad \phi \in C\left([-1,0], R^{2}\right) .
$$

In fact, we can choose

$$
\eta(\theta, \mu)= \begin{cases}\left(\tau_{20}^{*}+\mu\right)\left(A^{\prime}+B^{\prime}+C^{\prime}\right), & \theta=0, \\ \left(\tau_{20}^{*}+\mu\right)\left(B^{\prime}+C^{\prime}\right), & \theta \in\left[-\frac{\tau_{1 *}}{\tau_{20}^{*}}, 0\right), \\ \left(\tau_{20}^{*}+\mu\right) C^{\prime}, & \theta \in\left(-1,-\frac{\tau_{1 *}}{\tau_{20}^{*}}\right), \\ 0, & \theta=-1 .\end{cases}
$$

For $\phi \in C([-1,0])$, we define

$$
A(\mu) \phi= \begin{cases}\frac{d \phi(\theta)}{d \theta}, & -1 \leq \theta<0 \\ \int_{-1}^{0} d \eta(\theta, \mu) \phi(\theta), & \theta=0\end{cases}
$$

and

$$
R(\mu) \phi= \begin{cases}0, & -1 \leq \theta<0 \\ F(\mu, \phi), & \theta=0\end{cases}
$$

Then system (66) can be transformed into the following operator equation:

$$
\dot{u}(t)=A(\mu) u_{t}+R(\mu) u_{t},
$$

where $u_{t}=u(t+\theta)=\left(u_{1}(t+\theta), u_{2}(t+\theta)\right)$ for $\theta \in[0,-1]$.

For $\varphi \in C^{1}\left([0,1],\left(R^{2}\right)^{*}\right)$, we define the adjoint operator $A^{*}$ of $A$ :

$$
A^{*}(\varphi)= \begin{cases}-\frac{d \varphi(s)}{d s}, & 0<s \leq 1, \\ \int_{-1}^{0} d \eta^{T}(s, \mu) \varphi(-s), & s=0\end{cases}
$$

and a bilinear inner product:

$$
\langle\varphi(s), \phi(\theta)\rangle=\bar{\varphi}^{T}(0) \phi(0)-\int_{\theta=-1}^{0} \int_{\xi=0}^{\theta} \bar{\varphi}^{T}(\xi-\theta) d \eta(\theta) \phi(\xi) d \xi,
$$

where $\eta(\theta)=\eta(\theta, 0)$.

Let $\rho(\theta)=\left(1, \rho_{2}\right)^{T} e^{i \omega_{2}^{*} \tau_{20}^{*} \theta}$ be the eigenvectors of $A(0)$ corresponding to $i \omega_{2}^{*} \tau_{20}^{*}$ and $\rho^{*}(s)=D\left(1, \rho_{2}^{*}\right) e^{i \omega_{2}^{*} \tau_{20}^{*} s}$ be the eigenvectors of $A^{*}$ corresponding to $-i \omega_{2}^{*} \tau_{20}^{*}$. By a simple computation, we can get

$$
\rho_{2}=\frac{c_{21}}{i \omega_{2}^{*}-c_{22} e^{-i \tau_{20}^{*} \omega_{2}^{*}}}, \quad \rho_{2}^{*}=-\frac{i \omega_{2}^{*}+c_{22} e^{i \tau_{20}^{*}} \omega_{2}^{*}}{a_{11}+b_{11} e^{i \tau_{1 *}} \omega_{2}^{*}} .
$$

From Eq. (73), we choose

$$
\bar{D}=\left[1+\rho_{2}+\rho_{2}^{*}+\tau_{1 *} b_{11} e^{-i \tau_{1 *} \omega_{2}^{*}}+\bar{\rho}_{2}^{*} \tau_{20}^{*} e^{-i \tau_{20}^{*} \omega_{2}^{*}}\left(c_{21}+c_{32} \rho_{2}\right)\right]^{-1}
$$

such that $\left\langle\rho^{*}, \rho\right\rangle=1,\left\langle\rho^{*}, \bar{\rho}\right\rangle=0$. 
In the remainder of this section, following the algorithms given in [21] and using a similar computation process to that in [23], we obtain the following coefficients that determine the properties of the Hopf bifurcation:

$$
\begin{aligned}
g_{20}= & 2 \tau_{20}^{*} \bar{D}\left[g_{1}+g_{2} \rho^{(2)}(0)+g_{3}\left(\rho^{(2)}(0)\right)^{2}+g_{4} \rho^{(1)}\left(-\frac{\tau_{1 *}}{\tau_{20}^{*}}\right)+\bar{\rho}_{2}^{*}\left(h_{1}\left(\rho^{(1)}(-1)\right)^{2}\right.\right. \\
& \left.\left.+h_{2} \rho^{(1)}(-1) \rho^{(2)}(0)+h_{3} \rho^{(1)}(-1) \rho^{(2)}(-1)\right)\right], \\
g_{11}= & \tau_{20}^{*} \bar{D}\left[2 g_{1}+g_{2}\left(\bar{\rho}^{(2)}(0)+\rho^{(2)}(0)\right)+2 g_{3} \rho^{(2)}(0) \bar{\rho}^{(2)}(0)+g_{4}\left(\bar{\rho}^{(1)}\left(-\frac{\tau_{1 *}}{\tau_{20}^{*}}\right)\right.\right. \\
& \left.+\rho^{(1)}\left(-\frac{\tau_{1 *}}{\tau_{20}^{*}}\right)\right)+\bar{\rho}_{2}^{*}\left(2 h_{1} \rho^{(1)}(-1) \rho^{(1)}(-1)+h_{2}\left(\rho^{(1)}(-1) \bar{\rho}^{(2)}(0)\right.\right. \\
& \left.\left.\left.+\bar{\rho}^{(1)}(-1) \rho^{(2)}(0)\right)+h_{3}\left(\rho^{(1)}(-1) \bar{\rho}^{(2)}(-1)+\bar{\rho}^{(1)}(-1) \rho^{(2)}(-1)\right)\right)\right] \\
g_{02}= & 2 \tau_{20}^{*} \bar{D}\left[g_{1}+g_{2} \bar{\rho}^{(2)}(0)+g_{3}\left(\bar{\rho}^{(2)}(0)\right)^{2}+g_{4} \bar{\rho}^{(1)}\left(-\frac{\tau_{1 *}}{\tau_{20}^{*}}\right)+\bar{\rho}_{2}^{*}\left(h_{1}\left(\bar{\rho}^{(1)}(-1)\right)^{2}\right.\right. \\
& \left.\left.+h_{2} \bar{\rho}^{(1)}(-1) \bar{\rho}^{(2)}(0)+h_{3} \bar{\rho}^{(1)}(-1) \bar{\rho}^{(2)}(-1)\right)\right] \\
g_{21}= & 2 \tau_{20}^{*} \bar{D}\left[g_{1}\left(W_{11}^{(1)}(0)+W_{20}^{(1)}(0)\right)+g_{2}\left(W_{11}^{(1)}(0) \rho^{(2)}(0)+\frac{1}{2} W_{20}^{(1)}(0) \bar{\rho}^{(2)}(0)\right.\right. \\
& \left.\left.\left.+2 \rho^{(1)}(-1) \rho^{(2)}(-1) \bar{\rho}^{(1)}(-1)\right)+3 h_{6}\left(\rho^{(1)}(-1)\right)^{2} \bar{\rho}^{(1)}(-1)\right)\right] \\
& \left.+W_{11}^{(2)}(0)+\frac{1}{2} W_{20}^{(2)}(0)\right)+g_{3}\left(2 W_{11}^{(1)}(0) \rho^{(2)}(0)+W_{20}^{(2)}(0) \bar{\rho}^{(2)}(0)\right) \\
& +g_{4}\left(W_{11}^{(1)}(0) \rho^{(1)}\left(-\frac{\tau_{1 *}}{\tau_{20}^{*}}\right)+\frac{1}{2} W_{20}^{(1)}(0) \bar{\rho}^{(1)}\left(-\frac{\tau_{1 *}}{\tau_{20}^{*}}\right)\right. \\
& +h_{2}\left(W_{11}^{(1)}(-1) \rho^{(2)}(0)+\frac{1}{2} W_{20}^{(1)}(-1) \bar{\rho}^{(2)}(0)+W_{11}^{(2)}(0) \rho^{(1)}(-1)\right. \\
& \left.+W_{11}^{(1)}\left(-\frac{\tau_{1 *}}{\tau_{20}^{*}}\right)+\frac{1}{2} W_{20}^{(1)}\left(-\frac{\tau_{1 *}}{\tau_{20}^{*}}\right)\right) \\
& +g_{5}\left(\bar{\rho}^{(2)}(0)+2 \rho^{(2)}(0)\right)+g_{6}\left(\left(\rho^{(2)}(0)\right)^{2}+2 \rho^{(2)}(0) \bar{\rho}^{(2)}(0)\right)+3 g_{7} \\
& +3 g_{8}\left(\rho^{(2)}(0)\right)^{2} \bar{\rho}^{(2)}(0)+\bar{\rho}_{2}^{*}\left(h _ { 1 } \left(2 W_{11}^{(1)}(-1) \rho^{(1)}(-1)+W_{11}^{(1)}(-1) \rho^{(2)}(-1)+\frac{1}{2} W_{20}^{(1)}(-1) \bar{\rho}^{(2)}(-1)\right.\right. \\
& \\
& \\
& \\
&
\end{aligned}
$$

with

$$
\begin{aligned}
& W_{20}(\theta)=\frac{i g_{20} \rho(0)}{\tau_{20}^{*} \omega_{2}^{*}} e^{i \tau_{20}^{*} \omega_{2}^{*} \theta}+\frac{i \bar{g}_{02} \bar{\rho}(0)}{3 \tau_{20}^{*} \omega_{2}^{*}} e^{-i \tau_{20}^{*} \omega_{2}^{*} \theta}+E_{20} e^{2 i \tau_{20}^{*} \omega_{2}^{*} \theta}, \\
& W_{11}(\theta)=-\frac{i g_{11} \rho(0)}{\tau_{20}^{*} \omega_{2}^{*}} e^{i \tau_{20} \omega_{2}^{*} \theta}+\frac{i \bar{g}_{11} \bar{\rho}(0)}{\tau_{20}^{*} \omega_{2}^{*}} e^{-i \tau_{20}^{*} \omega_{2}^{*} \theta}+E_{11},
\end{aligned}
$$


where $E_{20}$ and $E_{11}$ can be computed by the following equations, respectively:

$$
\begin{aligned}
& E_{20}=2\left(\begin{array}{cc}
2 i \omega_{2}^{*}-a_{11}-b_{11} e^{-2 i \tau_{1 *} \omega_{2}^{*}} & -a_{12} \\
-c_{21} e^{-2 i \tau_{20}^{*} \omega_{2}^{*}} & 2 i \omega_{2}^{*}-c_{22} e^{-2 i \tau_{20}^{*} \omega_{2}^{*}}
\end{array}\right)^{-1} \times\left(\begin{array}{l}
E_{20}^{(1)} \\
E_{20}^{(2)}
\end{array}\right), \\
& E_{11}=-\left(\begin{array}{cc}
a_{11}+b_{11} & a_{12} \\
c_{21} & c_{22}
\end{array}\right)^{-1} \times\left(\begin{array}{c}
E_{11}^{(1)} \\
E_{11}^{(2)}
\end{array}\right),
\end{aligned}
$$

with

$$
\begin{aligned}
E_{20}^{(1)}= & g_{1}+g_{2} \rho^{(2)}(0)+g_{3}\left(\rho^{(2)}(0)\right)^{2}+g_{4} \rho^{(1)}\left(-\frac{\tau_{1 *}}{\tau_{20}^{*}}\right), \\
E_{20}^{(2)}= & h_{1}\left(\rho^{(1)}(-1)\right)^{2}+h_{2} \rho^{(1)}(-1) \rho^{(2)}(0)+h_{3} \rho^{(1)}(-1) \rho^{(2)}(-1), \\
E_{11}^{(1)}= & 2 g_{1}+g_{2}\left(\bar{\rho}^{(2)}(0)+\rho^{(2)}(0)\right)+2 g_{3} \rho^{(2)}(0) \bar{\rho}^{(2)}(0) \\
& +g_{4}\left(\bar{\rho}^{(1)}\left(-\frac{\tau_{1 *}}{\tau_{20}^{*}}\right)+\rho^{(1)}\left(-\frac{\tau_{1 *}}{\tau_{20}^{*}}\right)\right), \\
E_{11}^{(2)}= & 2 h_{1} \rho^{(1)}(-1) \rho^{(1)}(-1)+h_{2}\left(\rho^{(1)}(-1) \bar{\rho}^{(2)}(0)+\bar{\rho}^{(1)}(-1) \rho^{(2)}(0)\right) \\
& +h_{3}\left(\rho^{(1)}(-1) \bar{\rho}^{(2)}(-1)+\bar{\rho}^{(1)}(-1) \rho^{(2)}(-1)\right) .
\end{aligned}
$$

Therefore, we can calculate the following values:

$$
\begin{aligned}
& C_{1}(0)=\frac{i}{2 \tau_{20}^{*} \omega_{2}^{*}}\left(g_{11} g_{20}-2\left|g_{11}\right|^{2}-\frac{\left|g_{02}\right|^{2}}{3}\right)+\frac{g_{21}}{2}, \\
& \mu_{2}=-\frac{\operatorname{Re}\left\{C_{1}(0)\right\}}{\operatorname{Re}\left\{\lambda^{\prime}\left(\tau_{20}^{*}\right)\right\}}, \\
& \beta_{2}=2 \operatorname{Re}\left\{C_{1}(0)\right\}, \\
& T_{2}=-\frac{\operatorname{Im}\left\{C_{1}(0)\right\}+\mu_{2} \operatorname{Im}\left\{\lambda^{\prime}\left(\tau_{20}^{*}\right)\right\}}{\tau_{20}^{*} \omega_{2}^{*}} .
\end{aligned}
$$

Based on the discussion above, we can obtain the following results for system (2).

Theorem 6 The direction of the Hopf bifurcation is determined by the sign of $\mu_{2}:$ if $\mu_{2}>0$ $\left(\mu_{2}<0\right)$, the Hopf bifurcation is supercritical (subcritical). The stability of bifurcating periodic solutions is determined by the sign of $\beta_{2}$ : if $\beta_{2}<0\left(\beta_{2}>0\right)$, the bifurcating periodic solutions are stable (unstable). The period of the bifurcating periodic solutions is determined by the sign of $T_{2}$ : if $T_{2}>0\left(T_{2}<0\right)$, the period of the bifurcating periodic solutions increases (decreases).

\section{Numerical example}

In order to verify the analytic results obtained above and depict the Hopf bifurcation phenomenon of system (2), we give some numerical simulations in this section. The study of system (2) in this paper is restricted only to a theoretical analysis, therefore, for the choice of the value of the parameters in system (2), we only consider the conditions mentioned in Section 2 and the simulation effect. We hope that it may be helpful for experimental studies of the real situation. To this end, we choose a set of parameters randomly which can 
Figure $1 E_{*}$ is stable for $\tau_{1}=2.35<\tau_{10}=2.7488$.
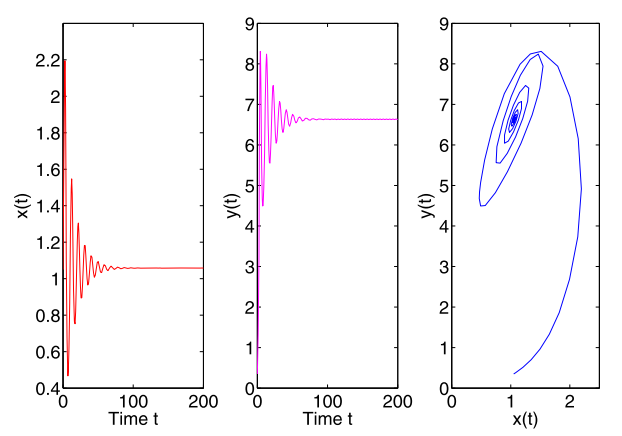

Figure $2 E_{*}$ is unstable for

$\tau_{1}=3.25>\tau_{10}=2.7488$.
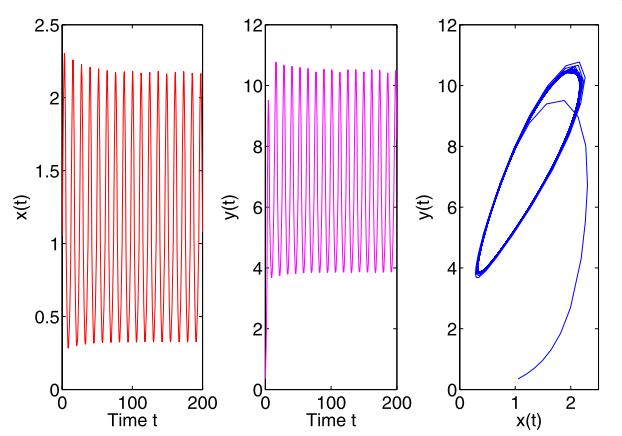

Figure $3 E_{*}$ is stable for $\tau_{2}=1.082<\tau_{20}=1.1705$.
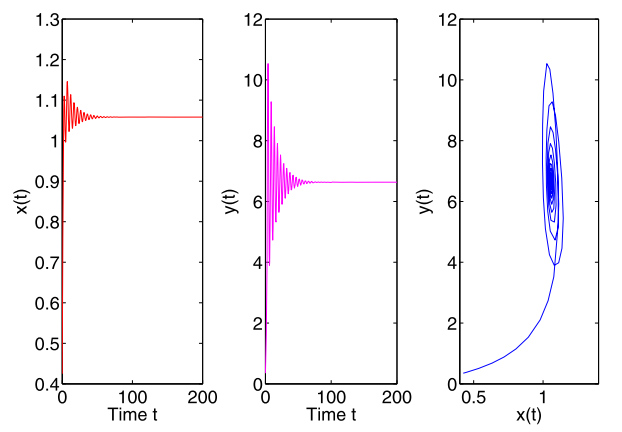

describe the Hopf bifurcation phenomenon of system (2) and get the following system:

$$
\left\{\begin{array}{l}
\frac{d x(t)}{d t}=\left(1-0.5 x\left(t-\tau_{1}\right)-\frac{0.2 y(t)}{1+0.15 x(t)+0.25 y(t)}\right) x(t), \\
\frac{d y(t)}{d t}=\left(1.2-\frac{0.3 y\left(t-\tau_{2}\right)}{x\left(t-\tau_{2}\right)+0.6}\right) y(t),
\end{array}\right.
$$

which has a positive equilibrium $E_{*}(1.0582,6.6328)$. Then we obtain $A_{10}=0.7513>0$, $A_{11}=1.7025>0$ and $C_{0}-D_{0}=-0.5185<0$. Thus, the conditions $\left(\mathrm{H}_{11}\right)$ and $\left(\mathrm{H}_{21}\right)$ holds.

For $\tau_{1}>0, \tau_{2}=0$. By a simple computation, we get $\omega_{10}=0.3848, \tau_{10}=2.7488$. From Theorem 1 , the positive equilibrium $E_{*}(1.0582,6.6328)$ of system $(105)$ is asymptotically stable when $\tau_{1}<\tau_{10}$. This is illustrated by Figure 1. As can be seen from Figure 1, when $\tau_{1}=2.35<\tau_{10}=2.7488$, the positive equilibrium $E_{*}(1.0582,6.6328)$ of system $(105)$ is asymptotically stable. Once $\tau_{1}$ passes through the critical value $\tau_{10}$, the positive equilibrium $E_{*}(1.0582,6.6328)$ of system (105) loses stability and a family of periodic solutions bifurcate from the positive equilibrium $E_{*}(1.0582,6.6328)$, which can be shown as in Fig- 


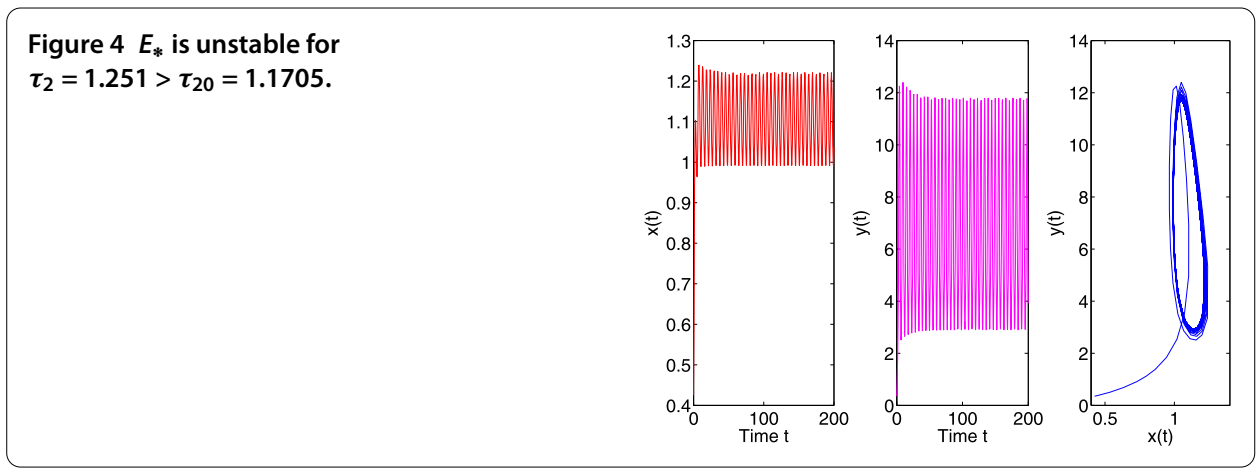

Figure $5 E_{*}$ is stable for $\tau=1.042<\tau_{0}=1.1062$.
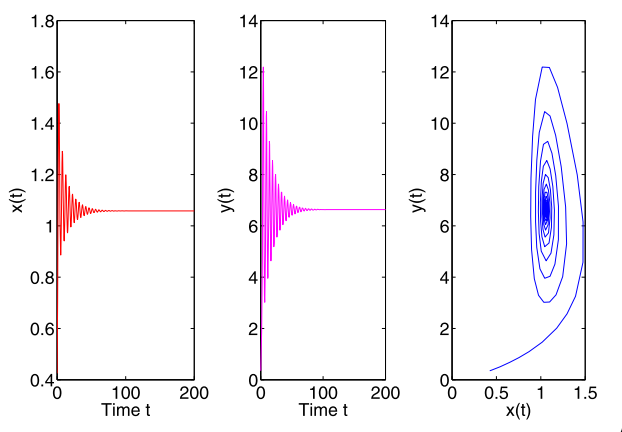

\section{Figure $6 E_{*}$ is unstable for} $\tau_{2}=1.163>\tau_{0}=1.1062$.
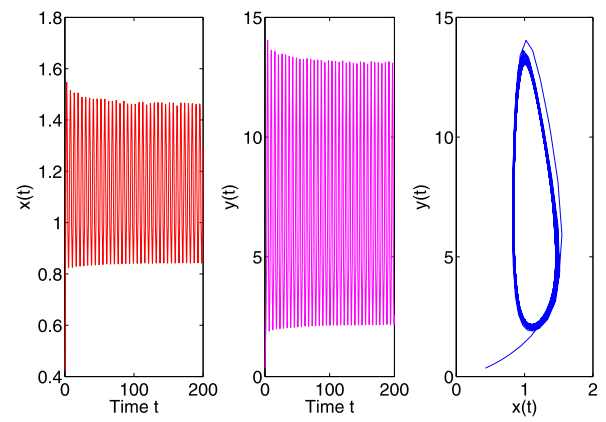

ure 2. As shown in Figure 2, we choose $\tau_{1}=3.25>\tau_{10}=2.7488$, then $E_{*}(1.0582,6.6328)$ is unstable and a Hopf bifurcation occurs and a family of periodic solutions bifurcate from $E_{*}(1.0582,6.6328)$. Similarly, we obtain $\omega_{20}=1.2455$ and $\tau_{20}=1.1705$ when $\tau_{1}=0$, $\tau_{2}>0$. According to Theorem 2 , the positive equilibrium $E_{*}(1.0582,6.6328)$ of system (105) is asymptotically stable when the value of $\tau_{2}$ is below the critical value $\tau_{20}$ and $E_{*}(1.0582,6.6328)$ becomes unstable when the value of $\tau_{2}$ is above the critical value $\tau_{20}$. This property is illustrated by Figures 3-4.

Consider the case $\tau_{1}=\tau_{2}=\tau>0$. By some complex computations, we obtain $\omega_{0}=$ 0.2754 and $\tau_{0}=1.1062$. From Theorem 3, we can conclude that the positive equilibrium $E_{*}(1.0582,6.6328)$ of system $(105)$ is asymptotically stable when $\tau \in[0,1.1062)$ and a Hopf bifurcation occurs when $\tau>1.1062=\tau_{0}$. Figure 5 shows that the positive equilibrium $E_{*}(1.0582,6.6328)$ of system (105) is asymptotically stable when $\tau=1.042 \in[0,1.1062)$. Then the positive equilibrium $E_{*}(1.0582,6.6328)$ of system (105) becomes unstable when $\tau=1.163>1.1062=\tau_{0}$, which can be shown in Figure 6 . 


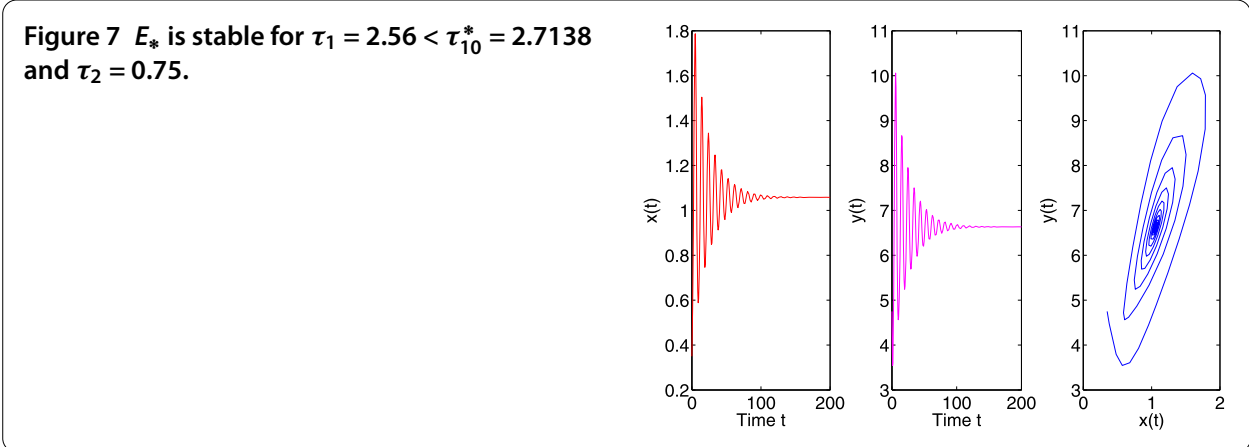

Figure $8 E_{*}$ is unstable for $\tau_{1}=3.015>\tau_{10}^{*}=2.7138$ and $\tau_{2}=0.75$.
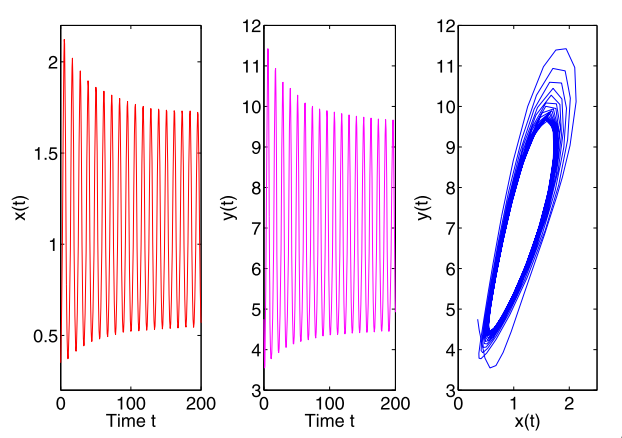

Figure $9 E_{*}$ is stable for $\tau_{2}=1.05<\tau_{20}^{*}=1.0988$ and $\tau_{1}=0.85$.
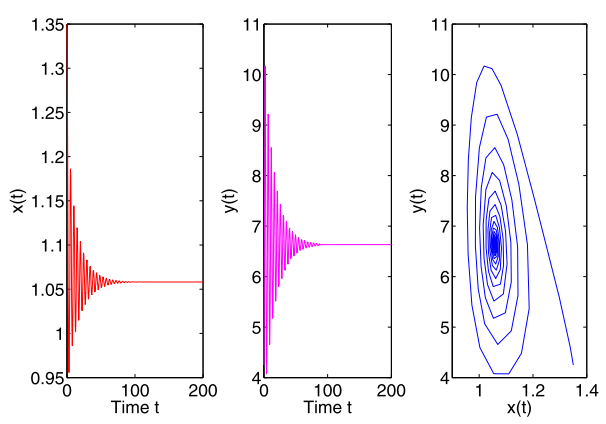

Now we consider $\tau_{1}>0, \tau_{2}=0.75 \in\left(0, \tau_{20}\right)$. We can obtain $\omega_{1}^{*}=1.2133$ and then we obtain $\tau_{10}^{*}=2.7138$. Figure 7 shows that the positive equilibrium $E_{*}(1.0582,6.6328)$ of system (24) is asymptotically stable when $\tau=2.56<2.7138=\tau_{10}^{*}$ and Figure 8 shows that there is a Hopf bifurcation occurs at the positive equilibrium $E_{*}(1.0582,6.6328)$ of system (105) and a family of periodic solutions bifurcate from $E_{*}(1.0582,6.6328)$ when $\tau=3.015>\tau_{10}^{*}=2.7138$. Similarly, we have $\omega_{2}^{*}=0.4950$ and $\tau_{20}^{*}=1.0988$ when $\tau_{2}>0$, $\tau_{1}=0.85 \in\left(0, \tau_{10}\right)$. The corresponding waveform and plots are shown in Figures 9-10. In addition, we obtain $\lambda^{\prime}\left(\tau_{20}^{*}\right)=3.9216-1.7717 i$ and $C_{1}(0)=-12.3787+9.7059 i$ by some complex computations. Further we have $\mu_{2}=3.1565>0, \beta_{2}=-24.7574<0, T_{2}=-7.5629<0$. According to Theorem 6, we can conclude that the Hopf bifurcation with respect to $\tau_{2}$ with $\tau_{1}=0.85 \in\left(0, \tau_{10}\right)$ is supercritical, the bifurcating periodic solutions are stable and decrease. Since the bifurcating periodic solutions of system (105) are stable, we know that the two species in system (105) can coexist in an oscillatory mode. 


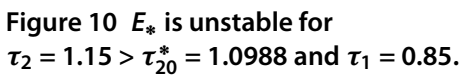


5. Liu, H, Liu, M, Wang, K, Wang, Y: Dynamics of a stochastic predator-prey system with Beddington-DeAngelis functional response. Appl. Math. Comput. 219, 2303-2312 (2012)

6. Liu, M, Wang, K: Global stability of stage-structured predator-prey models with Beddington-DeAngelis functional response. Commun. Nonlinear Sci. Numer. Simul. 16, 3791-3797 (2011)

7. Li, WL, Wang, LS: Stability and bifurcation of a delayed three-level food chain model with Beddington-DeAngelis functional response. Nonlinear Anal., Real World Appl. 10, 2471-2477 (2009)

8. Baek, H: Qualitative analysis of Beddington-DeAngelis type impulsive predator-prey models. Nonlinear Anal., Real World Appl. 11, 1312-1322 (2010)

9. Cui, JA, Takeuchi, Y: Permanence, extinction and periodic solution of predator-prey system with Beddington-DeAngelis functional response. J. Math. Anal. Appl. 317, 464-474 (2006)

10. Li, HY, Takeuchi, Y: Dynamics of the density dependent predator-prey system with Beddington-DeAngelis functional response. J. Math. Anal. Appl. 374, 644-654 (2011)

11. Liu, S, Beretta, E, Breda, D: Predator-prey model of Beddington-DeAngelis type with maturation and gestation delays. Nonlinear Anal., Real World Appl. 11, 4072-4091 (2010)

12. Yu, SB: Global stability of a modified Leslie-Gower model with Beddington-DeAngelis functional response. Adv. Differ Equ. 2014, 84 (2014)

13. Bianca, C, Ferrara, M, Guerrini, L: Qualitative analysis of a retarded mathematical framework with applications to living systems. Abstr. Appl. Anal. 2013, Article ID 7360528 (2013)

14. Jana, S, Kar, TK: Modeling and analysis of a prey-predator system with disease in the prey. Chaos Solitons Fractals 47, 42-53 (2009)

15. Bianca, C, Guerrini, L: On the Dalgaard-Strulik model with logistic population growth rate and delayed-carrying capacity. Acta Appl. Math. 128, 39-48 (2013)

16. Kar, TK, Ghorai, A: Dynamic behaviour of a delayed predator-prey model with harvesting. Appl. Math. Comput. 217, 9085-9104 (2011)

17. Cui, GH, Yan, XP: Stability and bifurcation analysis on a three-species food chain system with two delays. Commun. Nonlinear Sci. Numer. Simul. 16, 3704-3720 (2011)

18. Bianca, C, Guerrini, L: Existence of limit cycles in the Solow model with delayed-logistic population growth. Sci. World J. 2014, Article ID 207806 (2014)

19. Gakkhar, S, Singh, A: Complex dynamics in a prey predator system with multiple delays. Commun. Nonlinear Sci. Numer. Simul. 17, 914-929 (2012)

20. Bianca, C, Ferrara, M, Guerrini, L: The time delays' effects on the qualitative behavior of an economic growth model. Abstr. Appl. Anal. 2013, Article ID 901014 (2013)

21. Hassard, BD, Kazarinoff, ND, Wan, YH: Theory and Applications of Hopf Bifurcation. Cambridge University Press, Cambridge (1981)

22. Li, XL, Wei, JJ: On the zeros of a fourth degree exponential polynomial with applications to a neural network model with delays. Chaos Solitons Fractals 26, 519-526 (2005)

23. Bianca, C, Ferrara, M, Guerrini, L: The Cai model with time delay: existence of periodic solutions and asymptotic analysis. Appl. Math. Inf. Sci. 7, 21-27 (2013)

10.1186/1687-1847-2014-314

Cite this article as: Liu: Dynamical analysis of a delayed predator-prey system with modified Leslie-Gower and Beddington-DeAngelis functional response. Advances in Difference Equations 2014, 2014:314

\section{Submit your manuscript to a SpringerOpen ${ }^{\circ}$ journal and benefit from:}

- Convenient online submission

Rigorous peer review

- Immediate publication on acceptance

- Open access: articles freely available online

- High visibility within the field

- Retaining the copyright to your article 